\title{
The Aggregate Impact of Household Saving and Borrowing Constraints: Designing a Field Experiment in Uganda
}

\author{
Joseph P. Kaboski* Molly Lipscomb ${ }^{\dagger} \quad$ Virgiliu Midrigan ${ }^{\ddagger}$ \\ Online Appendix
}

*University of Notre Dame \& NBER, jkaboski@nd.edu.

$\dagger$ University of Virginia, ml4db@eservices.virginia.edu.

${ }_{\ddagger}^{\ddagger}$ New York University \& NBER, virgiliu.midrigan@nyu.edu. 
This appendix contains the details of the recursive formulation, computed policy functions, formulas for computing the returns to investment, calibration details including sensitivity analysis, and details of the dynamic response of variables to permanent and temporary relaxation of credit constraints..

\section{Recursive Formulation}

Because the quasi-hyperbolic problem involves a game between current and future selves, there are, in general multiple, equilibria and indeterminacy. Following Krusell, Kuruscu, and Smith (2002), we will focus on a particular set of equilibria: equilibria of Markovian strategies that are the limits of finite horizon problems. To solve for such equilibria, we write the problem recursively.

Let $V_{0}^{m}(b, e)$ be the value of an agent in the modern sector. Let $V_{0}^{\tau}(b, e)$ be the value of the agent in the traditional sector. Similarly, let $V^{m}(b, e)$ and $V^{\tau}(b, e)$ be the continuation values of future selves.

The Bellman equation is:

$$
V_{0}^{m}(b, e)=\max _{c, b^{\prime}} \frac{c^{1-\sigma}}{1-\sigma}+\beta \delta \int\left[(1-\pi) V^{m}\left(b^{\prime}, e\right)+\pi V^{\tau}\left(b^{\prime}, e^{\prime}\right)\right] d G\left(e^{\prime} \mid e\right)
$$

s.t.

$$
\begin{gathered}
c+(1+r) b=z_{m} e+b^{\prime} \\
b^{\prime} \leqslant \max (\gamma b, 0)+\lambda_{1}
\end{gathered}
$$

Similarly, we have:

$$
V_{0}^{\tau}(b, e)=\left\{\begin{array}{c}
\max \left[V_{0}^{\tau, m}(b, e), V_{0}^{\tau, \tau}(b, e)\right] \text { with prob. } \pi_{m} \\
V_{0}^{\tau, \tau}(b, e) \text { witj prob. }\left(1-\pi_{m}\right)
\end{array}\right.
$$

where $V_{0}^{\tau, m}(b, e)$ is the value of switching to the modern sector and $V_{0}^{\tau, \tau}(b, e)$ is the value of staying in the traditional sector and recall that $\pi_{m}$ is the probability that an investment opportunity arrives. We have

$$
V_{0}^{\tau, m}(b, e)=\max _{c, b^{\prime}} \frac{c^{1-\sigma}}{1-\sigma}+\beta \delta \int V^{m}\left(b^{\prime}, e^{\prime}\right) d G\left(e^{\prime} \mid e\right)
$$

s.t.

$$
\begin{gathered}
c+(1+r) b+\kappa=e+b^{\prime} \\
b^{\prime} \leqslant \max (\gamma b, 0)+\lambda_{1}+\lambda_{2} \kappa
\end{gathered}
$$

and

$$
V_{0}^{\tau, \tau}(b, e)=\max _{c, b^{\prime}} \frac{c^{1-\sigma}}{1-\sigma}+\beta \delta \int V^{\tau}\left(b^{\prime}, e^{\prime}\right) d G\left(e^{\prime} \mid e\right)
$$


s.t.

$$
\begin{aligned}
& c+(1+r) b=e+b^{\prime} \\
& b^{\prime} \leqslant \max (\gamma b, 0)+\lambda_{1}
\end{aligned}
$$

The solution to this problem gives decision rules $c^{m}(a, e), b^{\prime m}(b, e)$ for agents already in the modern sector, $c^{\tau, \tau}(b, e), b^{\prime \tau, \tau}(b, e)$ for agents that stay in the traditional sector, and $c^{\tau, m}(b, e), b^{\prime \tau, m}(b, e)$ for agents that switch to the modern sector. Moreover, we have a switching rule, $s(b, e)$ for agents in the traditional sector that receive an investment opportunity:

$$
s(b, e)=1 \text { if } V_{0}^{\tau, m}(b, e) \geqslant V_{0}^{\tau, \tau}(b, e)
$$

The continuation values of future selves are defined as follows:

$$
\begin{gathered}
V^{m}(b, e)=\frac{\tilde{c}^{m}(b, e)^{1-\sigma}}{1-\sigma}+\delta \int\left[(1-\pi) V^{m}\left(\tilde{b}^{\prime m}(b, e), e^{\prime}\right)+\pi V^{\tau}\left(\tilde{b}^{\prime m}(b, e), e^{\prime}\right)\right] d G\left(e^{\prime} \mid e\right) \\
V^{\tau}(b, e)=\left\{\begin{array}{c}
\tilde{s}(b, e) V^{\tau, m}(b, e)+(1-\tilde{s}(b, e)) V^{\tau, \tau}(b, e) \text { with prob. } \pi_{m} \\
V^{\tau, \tau}(b, e) \text { with prob. }\left(1-\pi_{m}\right) \\
V^{\tau, m}(b, e)=\frac{\tilde{c}^{\tau, m}(b, e)^{1-\sigma}}{1-\sigma}+\delta \int V^{m}\left(\tilde{b}^{\prime \tau, m}(b, e), e\right) d G\left(e^{\prime} \mid e\right) \\
V^{\tau, \tau}(b, e)=\frac{\tilde{c}^{\tau, \tau}(b, e)^{1-\sigma}}{1-\sigma}+\delta \int V^{\tau}\left(\tilde{b}^{\prime \tau, \tau}(b, e), e\right) d G\left(e^{\prime} \mid e\right)
\end{array}\right.
\end{gathered}
$$

Note that there is no maximization in this last set expressions for the continuation values of future selves. We use instead the policy functions $\tilde{s}(a, e), \tilde{c}(a, e)$ and $\tilde{b}^{\prime}(b, e)$, which are the Markov reaction functions of future selves. Denoting these joint reaction functions as $\tilde{\Gamma}(a, e)$ and the counterpart policy functions of the current self as $\Gamma(a, e)$, the Markov Perfect equilibrium is the solution of the fixed point problem, $\Gamma=\tilde{\Gamma}$.

\section{Policy Functions}

Figure 1.A reports the decision rules of a household with a relatively high level of productivity. The left column of the figure illustrates the savings rule, $a^{\prime}=-b^{\prime}$, for agents residing in the modern and traditional sectors, respectively, as a function of the agents' beginning of period assets. The right column illustrates the associated consumption choices. Superimposed on the savings rules is the 45-degree line: whenever the savings choice is above the 45-degree line, the agent increases its stock of savings: the intersection of the two lines thus shows the steady-state level of assets that would prevail absent any changes in productivity. Finally, the vertical line in the lower panels of the figures shows the switching cutoff: the minimum level of assets at which, given the opportunity to do so, the agent pays the fixed cost and 
joins the modern sector.

The upper panel of the figure is typical for Bewley-Aiyagari models of the type we study here: the consumption function is concave, poor agents save for precautionary reasons, and rich agents dissave because of impatience. The lower panel of the figure shows that the agent must have accumulated a sufficiently large level of savings to find it worthwhile to join the modern sector. When they do switch, their level of financial savings goes to 0 (the assumed borrowing limit in this example) and consumption experiences a discrete fall. Notice that in this particular example the agent's savings choice when poor is above the 45 degree line: the agent thus builds up a stock of asset and eventually switches to the modern sector.

Figure 2.A contrasts the decision rules of the relatively productive agent in Figure 1 with those of a relatively unproductive agent and focuses on the savings choices of those in the traditional sector. Notice that the cutoff level of assets necessary to switch to the modern sector is higher for the less productive agent. Moreover, less productive agents save less. Indeed, an agent that always receives the lower productivity realization does not save enough to build up the stock necessary to enter the modern sector (the 45-degree line intersects the savings rule before the cutoff is reached) and stays permanently in the traditional sector. Figure 3.A summarizes this discussion by illustrating the time-series paths of consumption and savings for the productive and unproductive agents. The more productive agents builds up the necessary stock of savings in 5 periods, switches to the modern sector and experiences a sharp increase in consumption after the initial investment period. The less productive agent, in contrast, saves little and never switches sectors. 


\section{Computing Returns to Investment}

We compute returns to (the long-lived, irreversible) investment in the model as a risk-neutral equivalent retunr. Assume a risk-neutral investor who faces an interest rate $r$. Such an investor is indifferent between adopting the modern sector and not doing so as long as the project has a net present value of zero. Let $V_{i}$ denote the expected present value of the output produced by the modern technology. This value satisfies:

$$
V_{i}=\left(z_{m}-1\right) e_{i}+\frac{(1-\pi)}{1+r} \sum_{j} P_{i, j} V_{j}
$$

where $P_{i, j}$ is the probability that a household with productivity $e_{i}$ in the current period draws productivity $e_{j}$ next period. Letting $V$ denote the vector of values for each productivity level, we have

$$
V(r)=\left(z_{m}-1\right)\left[I-\frac{1-\pi}{1+r} P\right]^{-1} e
$$

where $P$ is the matrix describing the Markov transition probabilities and $e$ is vector of productivities. Since entering the modern sector requires paying a fixed cost $\kappa$ one period in advance, the interest rate that makes an investor indifferent between investing in a project of type $i$ solves:

$$
\frac{1}{1+r_{i}} V_{i}\left(r_{i}\right)-\kappa=0
$$

We compute the returns to investment as the weighted average of $r_{i}$, weighting each productivity type by the fraction of households of that particular type that switch to the modern sector in the ergodic steady state of the economy.

\section{Calibration Tables}

Table 1 summarizes the calibration results discussed in the paper, while Table 2 presents comparative static results to key endogenous parameters. These comparative static simulations underscore the identification in the model by illustrating how these parameters affect the moments we target. First, an increase the short-run discount factor, $\beta$, from 0.55 in our baseline to 0.65 causes agents to save too much now in this economy: more than doubling their financial savings, and increasing their physical assets by more than one-third relative to the data. Second, if we reduce the persistence of productivity from 0.75 to 0.5 , the selection effect becomes weaker and the gap between the consumption of agent in the modern and traditional sector falls (by 15 percentage points) along with fraction entering the modern sector and the return to investment. These last two moments are affected more directly, however, by a reduction in the productivity advantage of the modern sector, $z_{m}$ : Lowering this advantage from 1.17 to 1.085 lowers the fraction of agents adopting the modern technology by more than half and the returns to investment by more than two-thirds. Finally, eliminating the assumption that investment opportunities arrive infrequently (increasing $\pi_{m}$ 
from 0.3 to 1 ) increases somewhat the fraction of agents that adopt the modern technology. ${ }^{1}$

\section{Dynamics of Simulation}

Here we present more of the details of the response of the economy to unanticipated permanent and transitory changes in the borrowing constraint parameters. Figures 4 .A and 5.A show the aggregate impacts of permanent increases, while Figures 6.A and Figure 7.A elaborate on the results presented in Figure 1 of the paper by giving greater detail of the dynamic responses for variables beyond consumption and presenting the responses for geometric discounters as well.

\footnotetext{
${ }^{1}$ If we fix $\pi_{m}=1$ and rather reduce $z_{m}$ to match the fraction of agents in the modern sector, the returns to investment decline to about 10\%, thus much lower than the estimates of de Mel et. al (2008).
} 

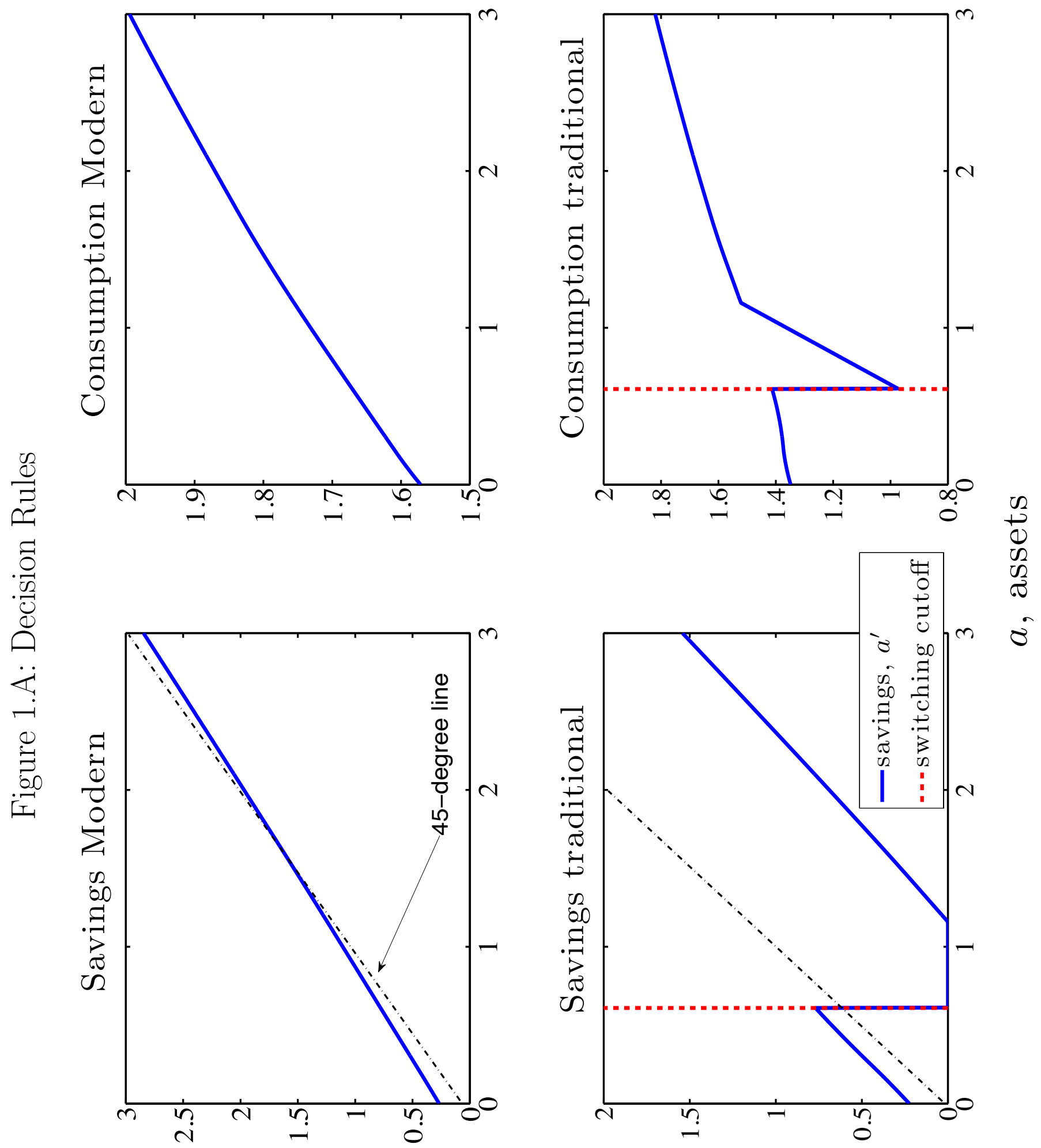


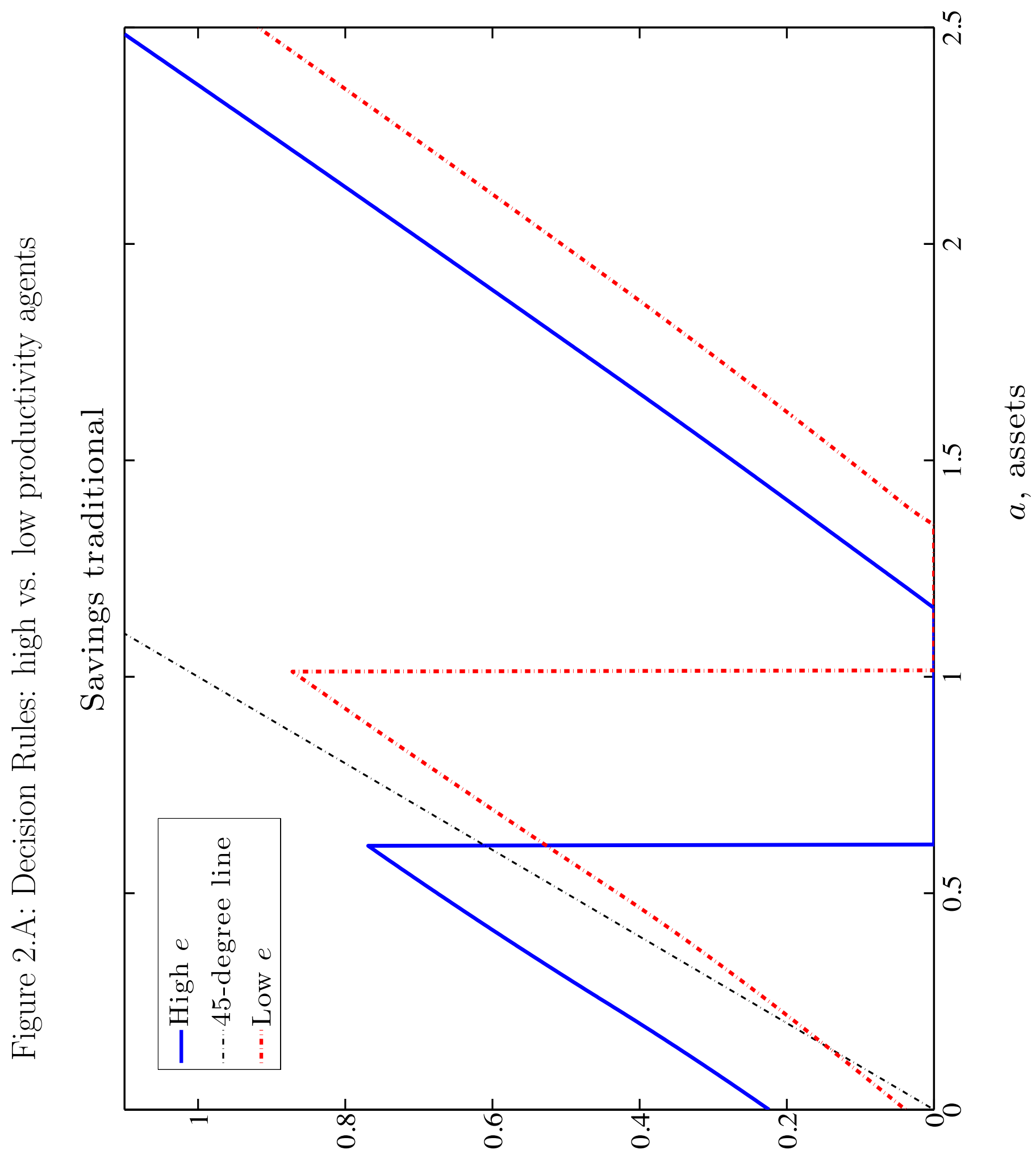




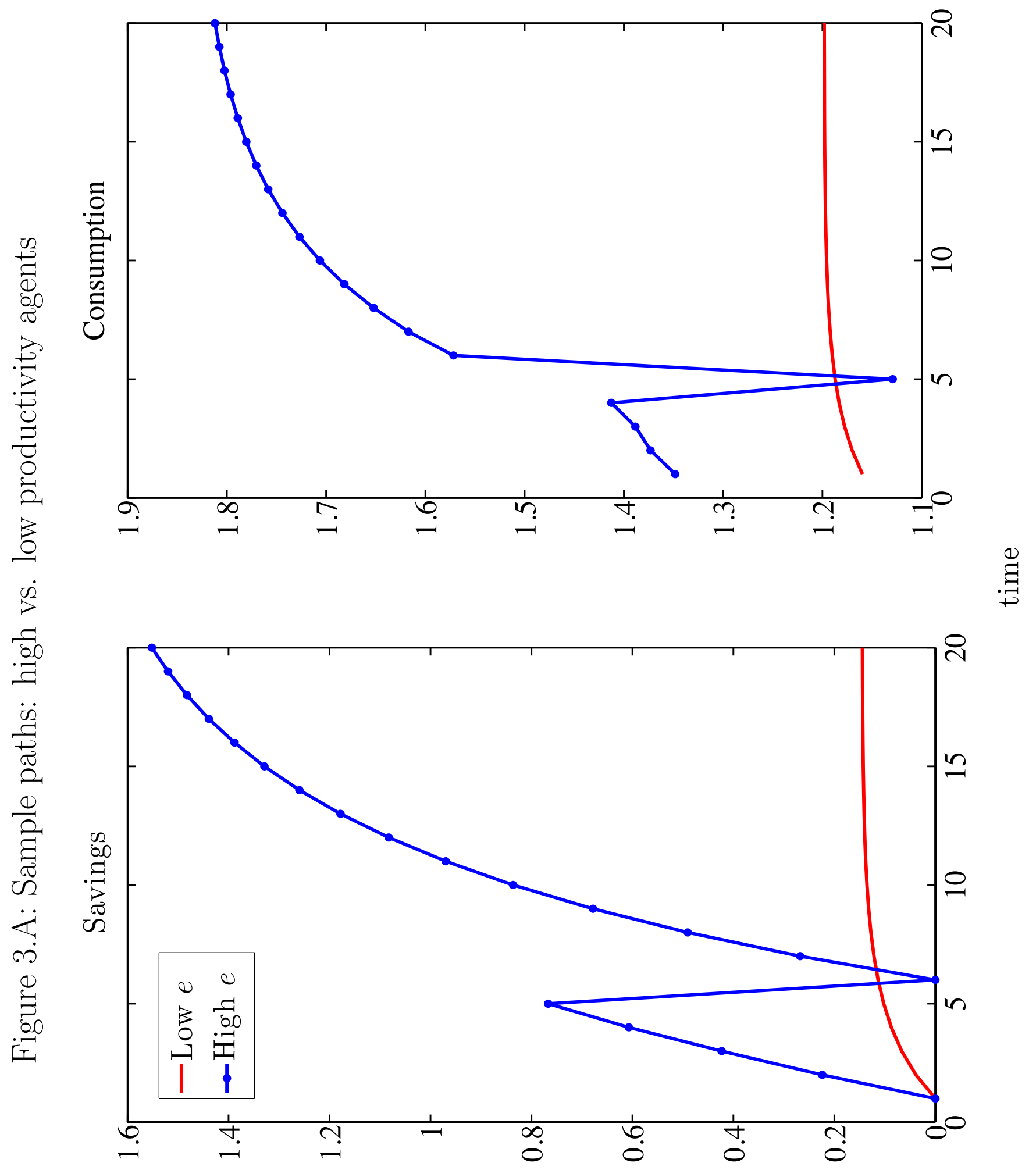




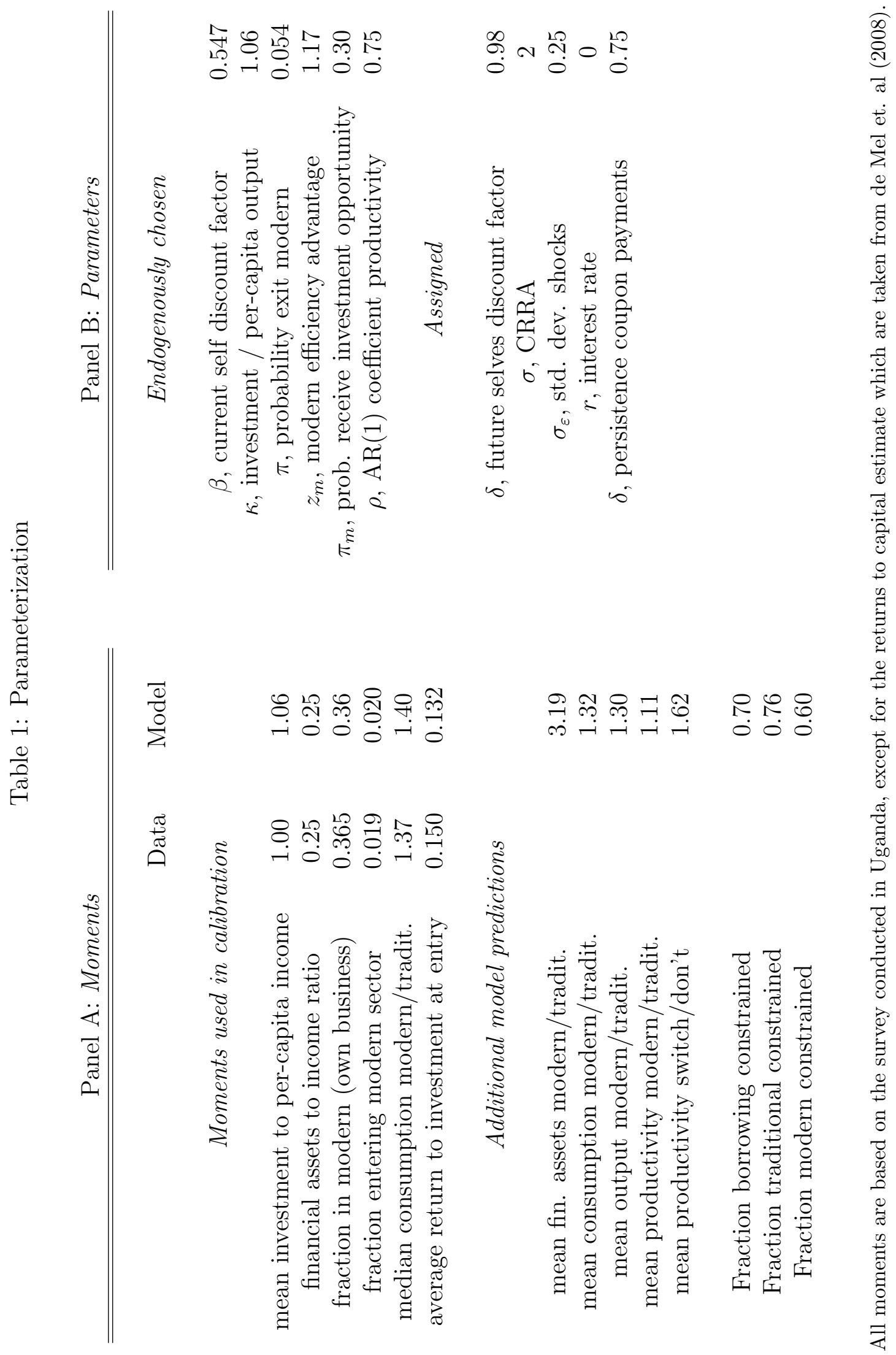




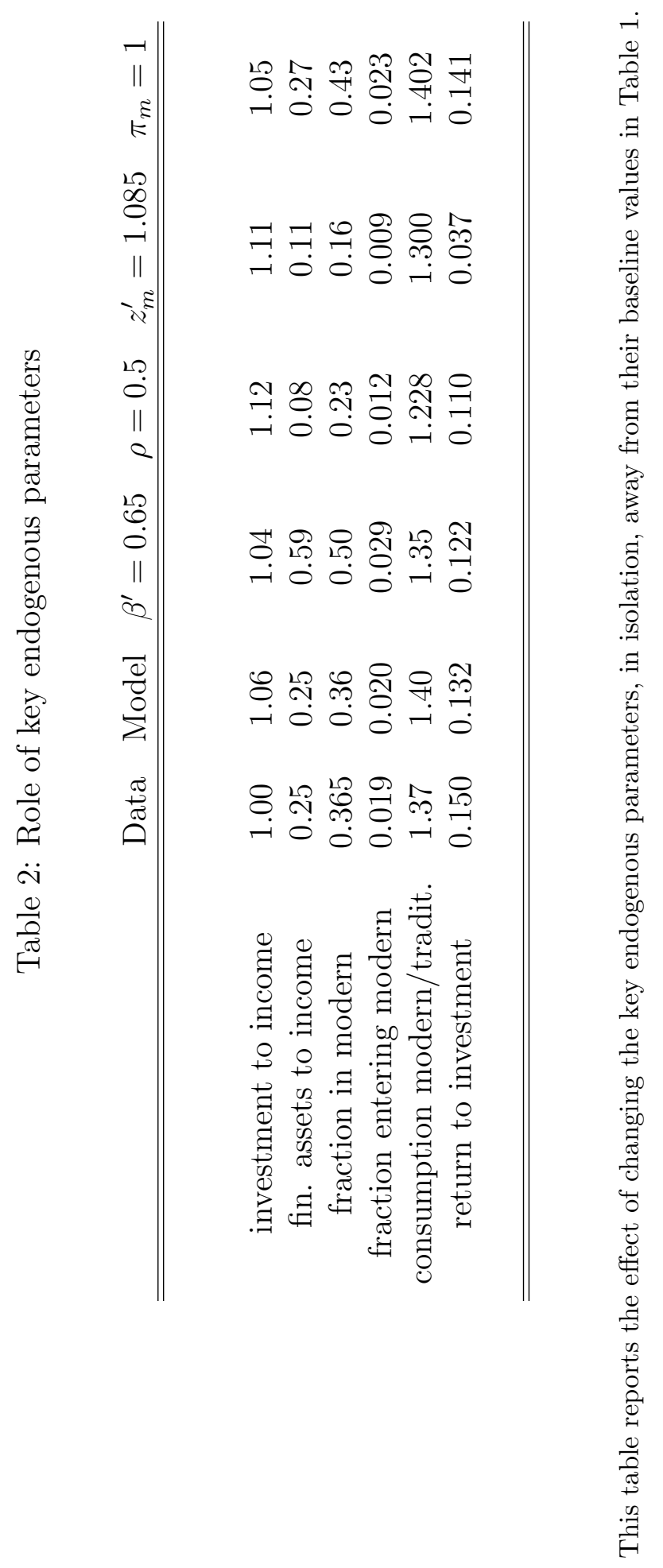



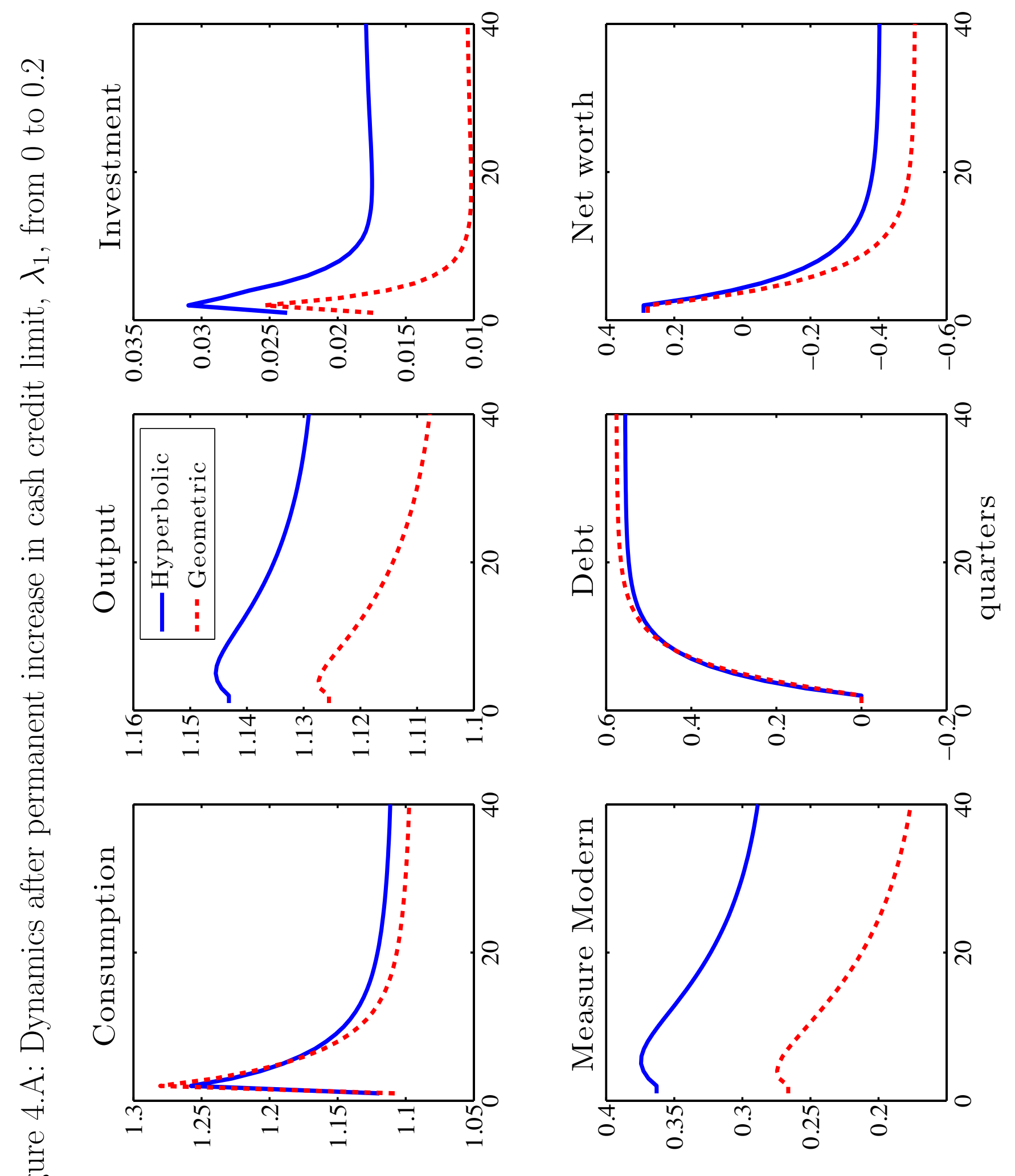

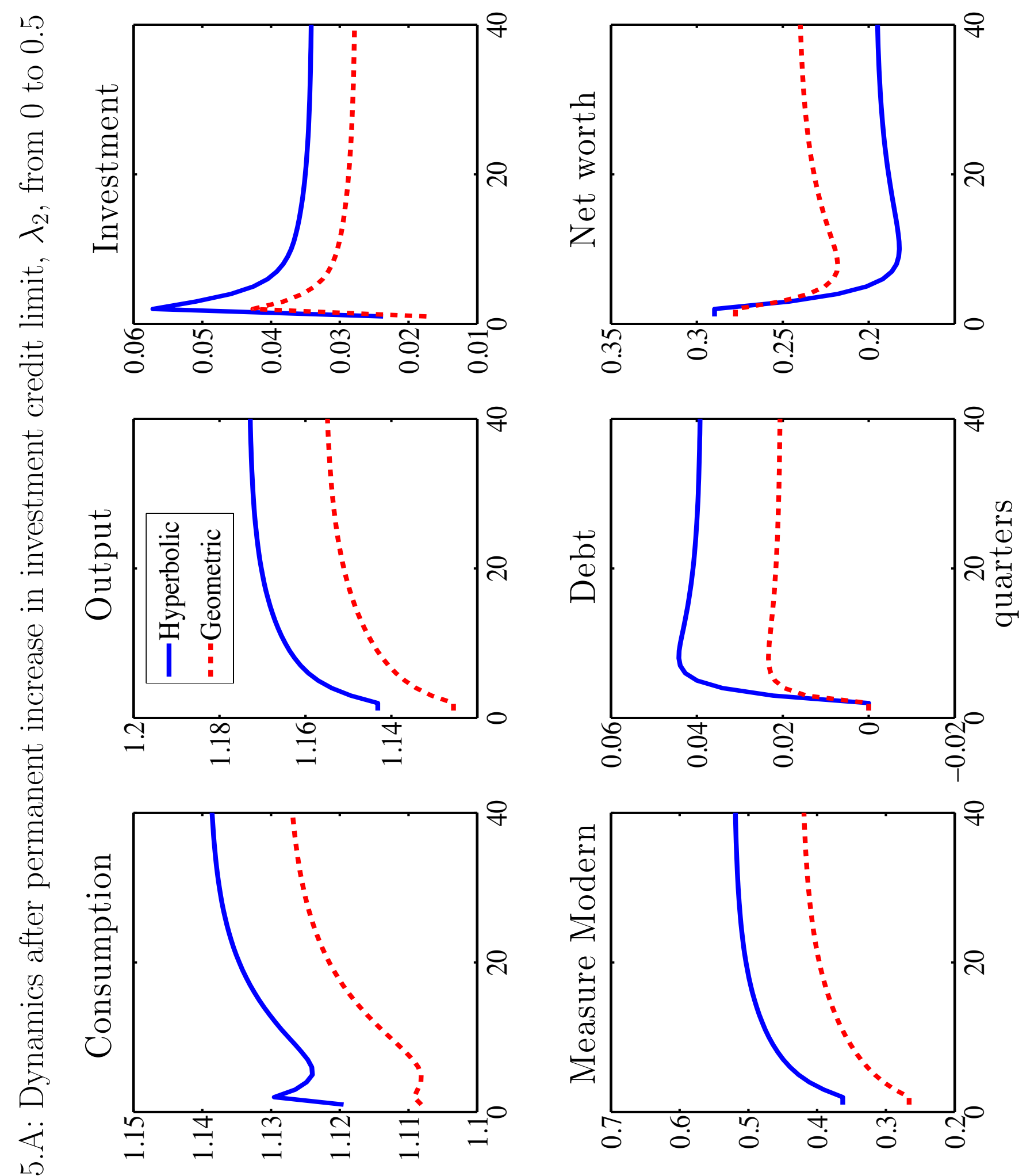

尚 

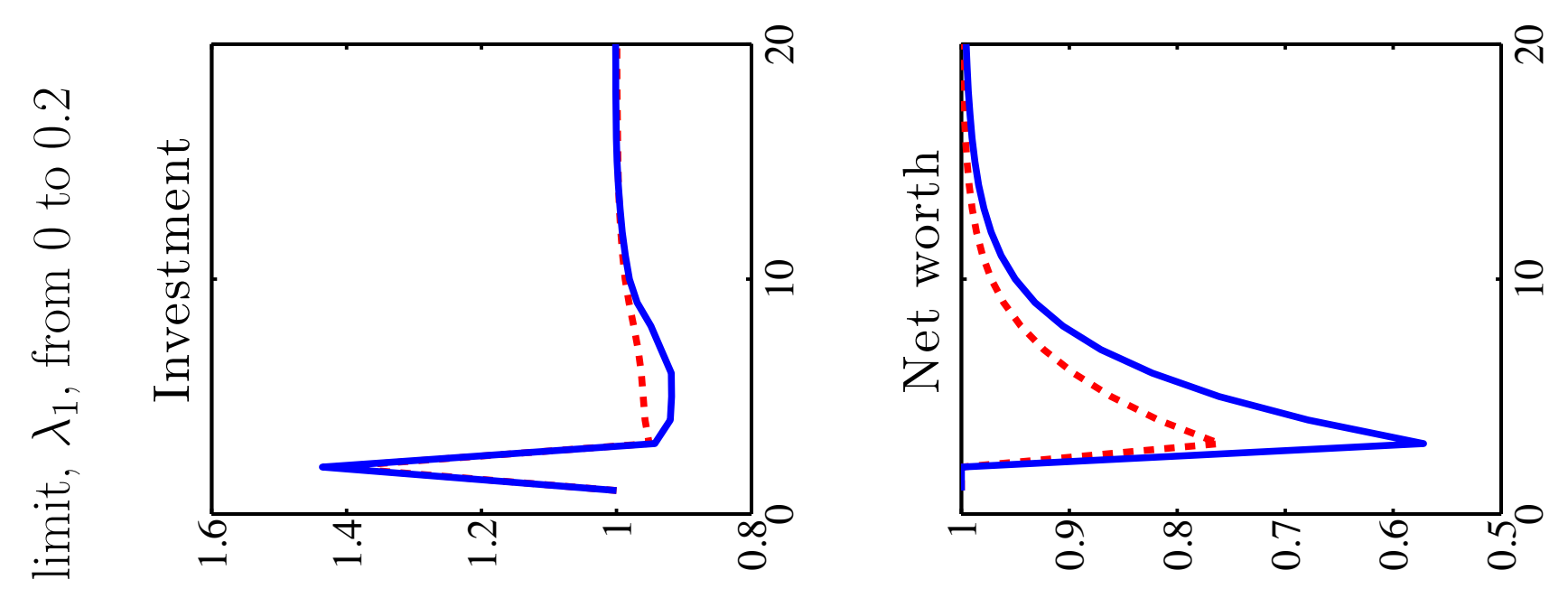

Ðٍ
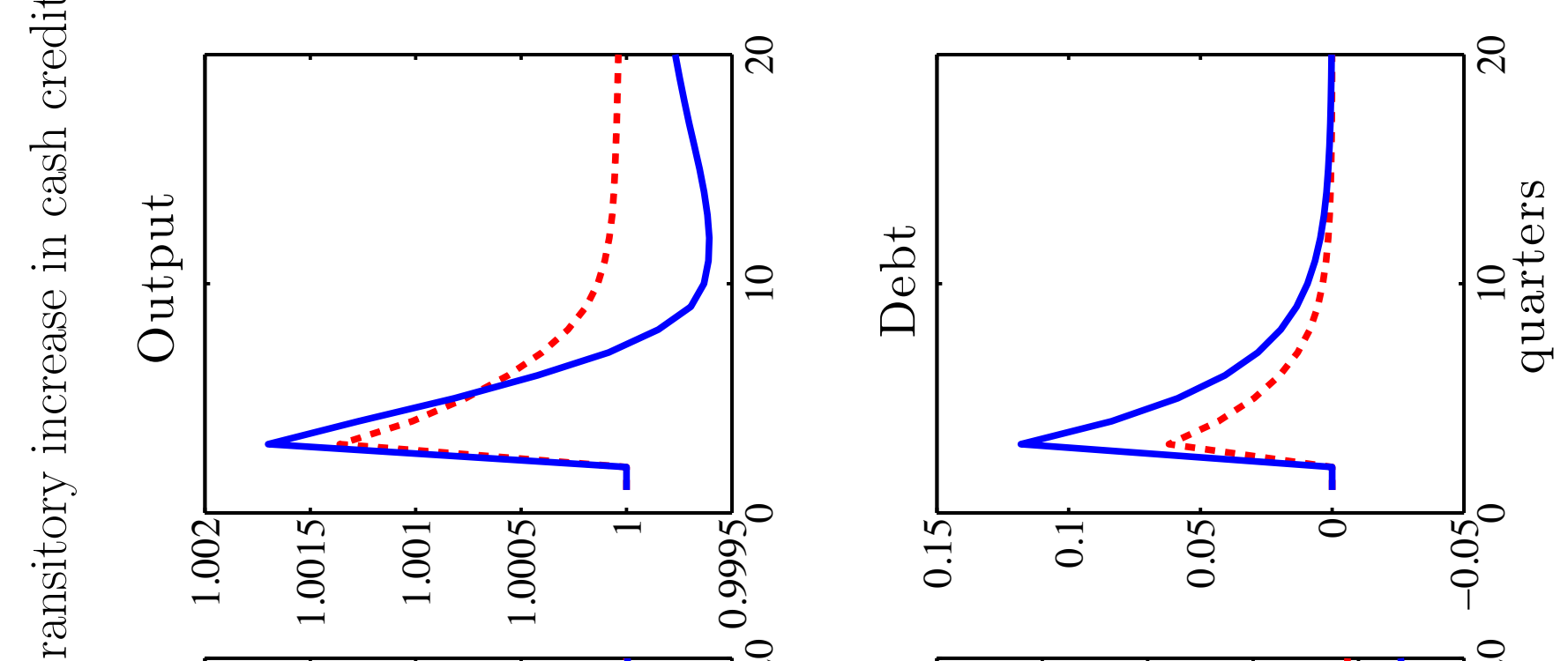

ช

도
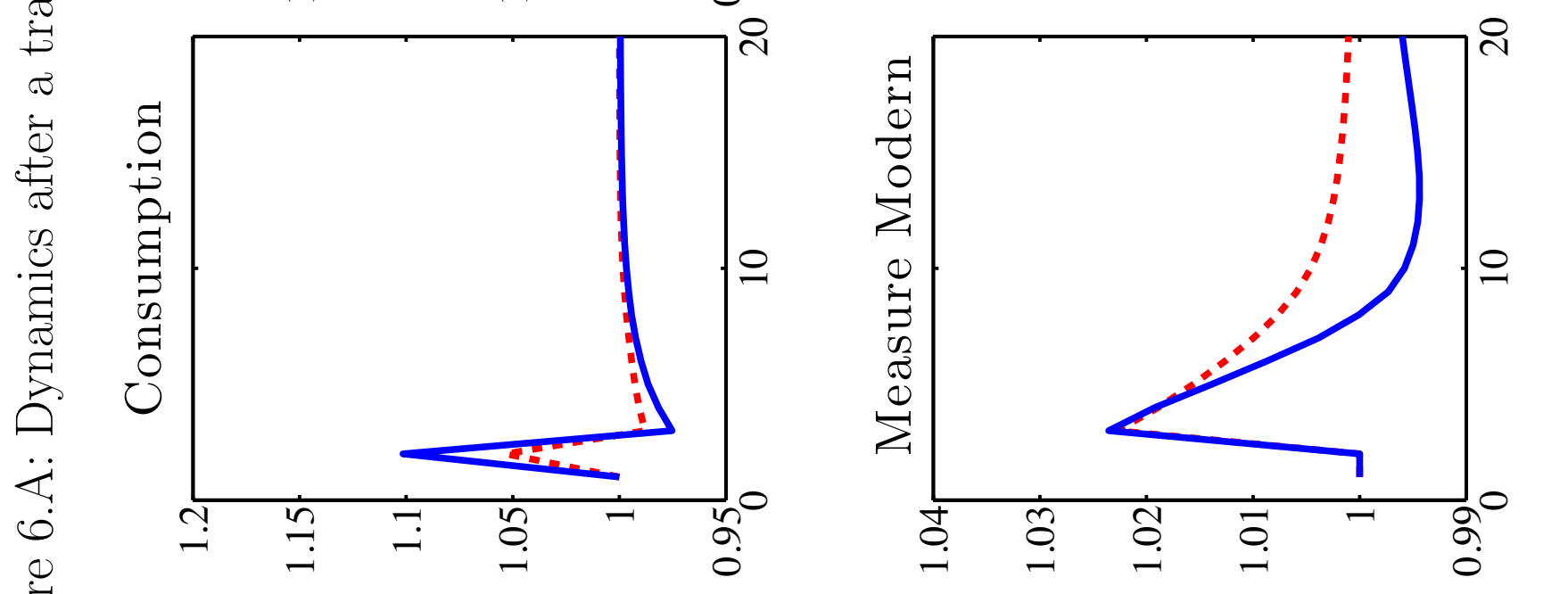

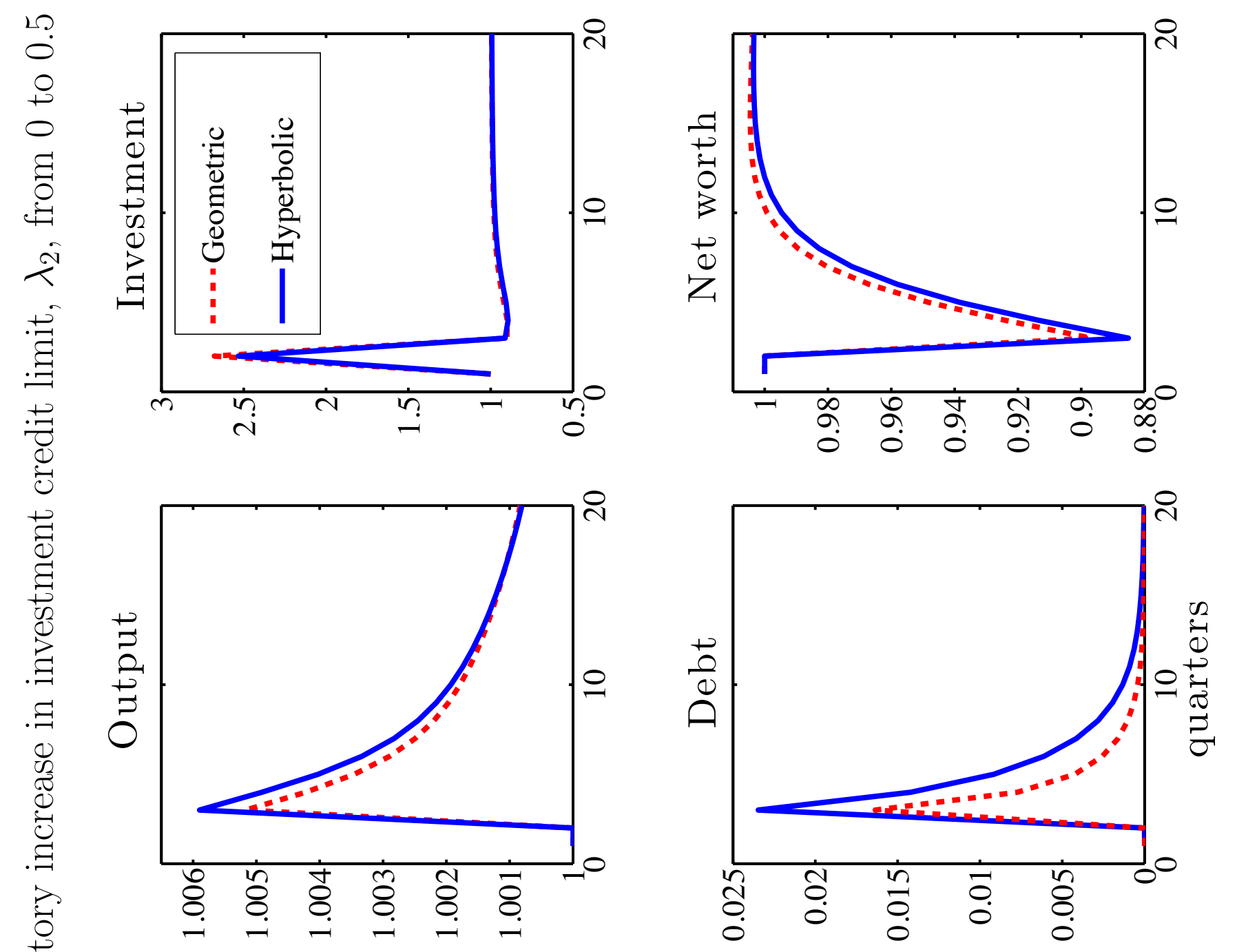

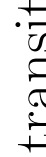
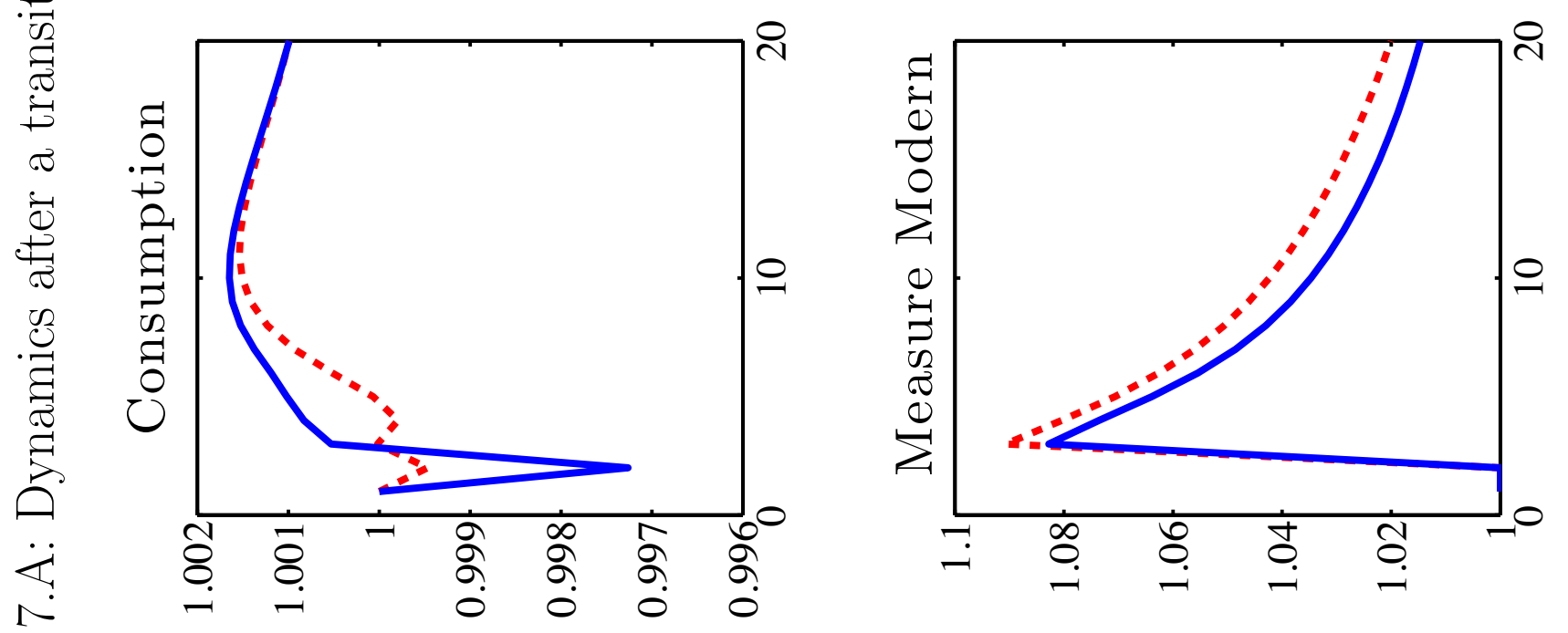

常 


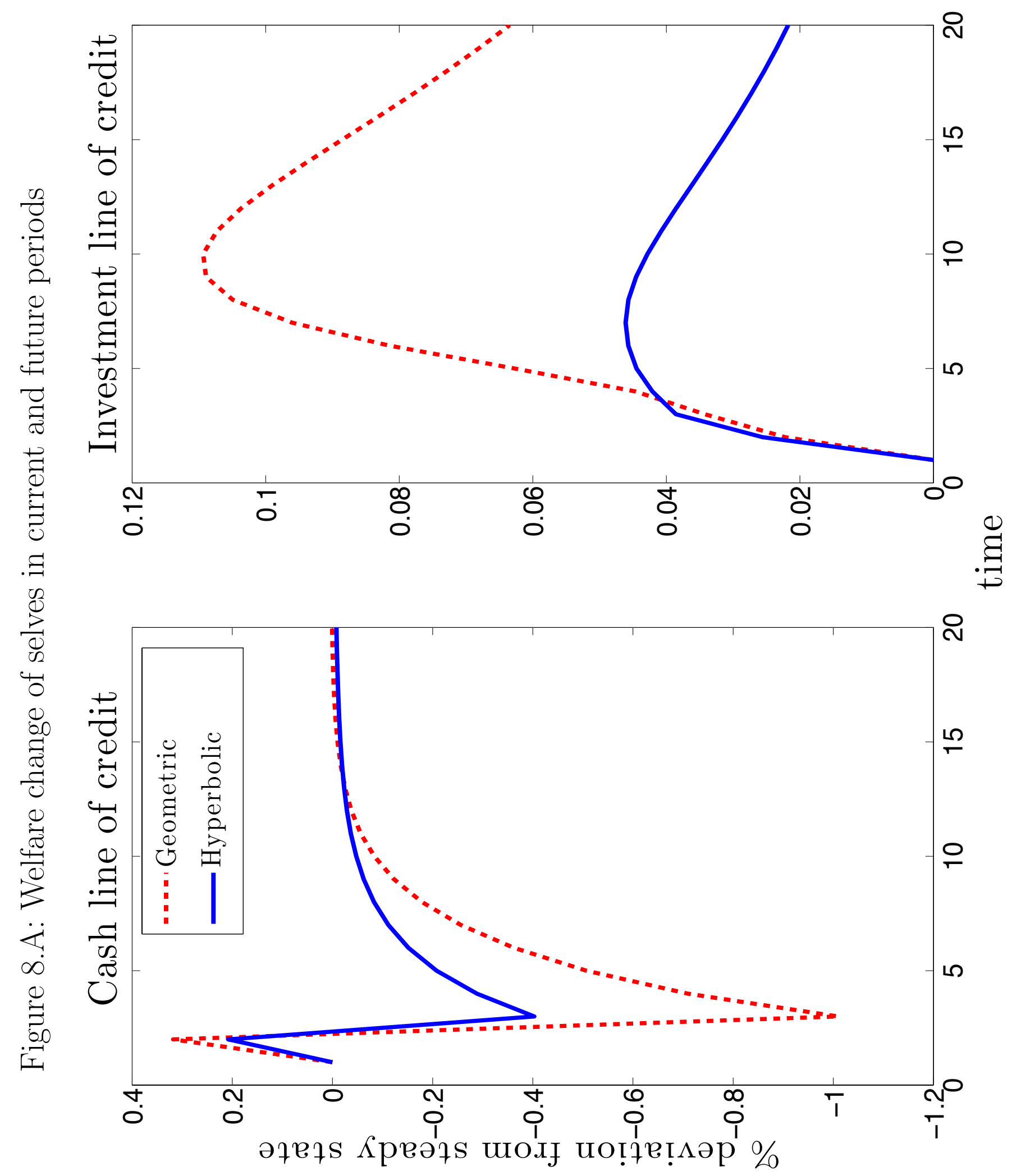

